Chapter 26

\title{
Phonon Generation and Detection by Single-Particle Tunneling in Superconductors*
}

\author{
W. Eisenmenger \\ III. Physikalisches Institut \\ der Universität Göttingen \\ Göttingen, West Germany
}

\section{INTRODUCTION}

In single-particle tunneling between superconductors energy from the voltage source is supplied to the tunneling junction in the form of quasiparticle excitations within the superconductors adjoining the tunneling barrier $\left({ }^{1,2}\right)$.

Simply speaking, the decay of these excitations is expected to produce heat. Since heat corresponds to incoherent phonon radiation with Planck's energy distribution (temperature well below Debye temperature), it is required that primary phonons directly emitted via electron-phonon interactions in quasiparticle decay undergo further phonon-electron and phonon-phonon interactions until the phonon energy distribution arrives at thermal equilibrium.

By proper experimental conditions it might be possible to prevent thermalization of primary decay phonons and to observe properties of their energy distribution.

\section{EXPECTED ENERGY DISTRIBUTION OF DECAY PHONONS}

According to Tewordt $\left({ }^{3,4}\right)$ deexcitation occurs in two steps predominantly via incoherent phonon emission. This is illustrated by the simple semiconductor model for tunneling between two identical superconductors

* Work underlying this chapter has been performed at Bell Telephone Laboratories, Murray Hill, N.J. in 1966 and 1967, on leave from the University of Göttingen. 


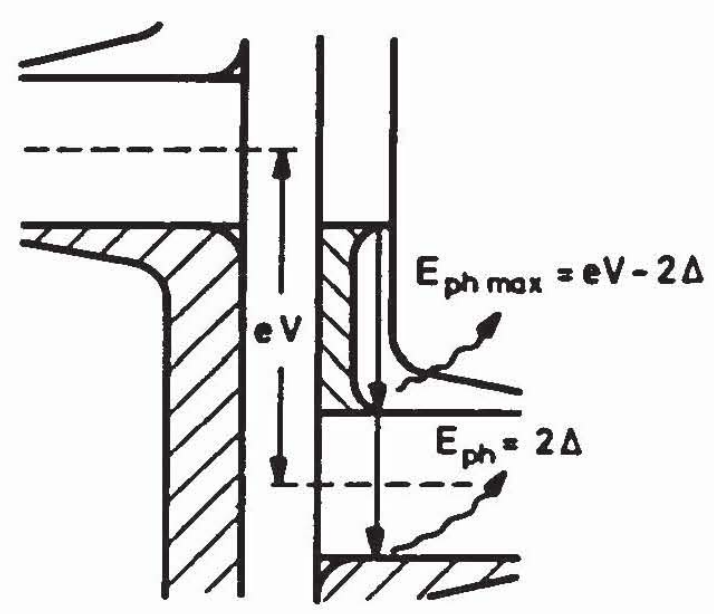

Fig. 1. Phonon generation, $e V \geq 2 \Delta$.

(Fig. 1). If a battery voltage $V>2 \Delta / e$ ( $2 \Delta$ is the energy gap, $e$ the electron charge) is applied to the junction of two identical superconductors, the energies of injected quasiparticles range from $\Delta$ to $\mathrm{eV}-\Delta$ with respect to the Fermi level. Relaxation occurs as a decay of quasiparticles to the upper gap edge via single-phonon emission, the continuous energy spectrum of relaxation phonons extending from 0 to $\mathrm{eV}-2 \Delta$. The succeeding recombination of quasiparticles to Cooper pairs results in recombination phonons of energy $2 \triangle$. Recombination phonon frequencies range from $10^{11}$ to $10^{12}$ cps depending on the superconducting energy gap. For tir as used in our phonon experiments, the frequency amounts to $2.8 \times 10^{11} \mathrm{cps}$, corresponding to an energy gap of $1.15 \mathrm{meV}$ at $1.2^{\circ} \mathrm{K}$. This frequency is about one order of magnitude higher than the main thermal phonon frequency at $1.2^{\circ} \mathrm{K}$.

Relaxation and recombination lifetimes are of the order of $10^{-9} \mathrm{sec}\left({ }^{5}\right)$ and $10^{-7} \sec \left({ }^{5,6}\right)$, respectively, indicating that the phonon spectra should be fairly well defined. Contributions of photon emission in relaxation $\left(^{3}\right)$ and recombination ( ${ }^{7}$ ) appear negligibly small compared to phonon emission.

For experiments concerning relaxation and recombination phonons it is necessary that these phonons are directly transmitted into a substrate with little further phonon interactions and finally received by a frequencyselective detector. For tunneling junctions in form of thin (1000 $\AA$ ) stripes evaporated on a sapphire crystal this condition is at least partly fulfilled for phonons propagating perpendicular to the metal-sapphire interface. Estimates of the phonon mean free path in normal metals with respect to the predominant electron interaction amount to $10^{-5}$ to $10^{-4} \mathrm{~cm}$ at low temperatures. This estimate is based on ultrasonic measurements in tin $\left(^{8}\right)$, which have been extrapolated according to theory $\left(^{(}\right)$to frequencies of $3 \times 10^{11} \mathrm{cps}$. Information on the phonon mean free path in sapphire can be 
obtained by heat conductivity measurements $\left({ }^{10}\right)$; a value of the order of $1 \mathrm{~cm}$ is indicated. In addition, heat pulse experiments at $4^{\circ} \mathrm{K}\left({ }^{11,12}\right)$ proved that phonons of $10^{11} \mathrm{cps}$ are propagating in sapphire with little attenuation. Finally, the expected incoherent phonon radiation need to be probed by a detector with frequency- or energy-selective properties. These requirements are, in principle met by a phonon quantum detector.

\section{PHONON DETECTION BY INTERACTION WITH COOPER PAIRS}

According to a suggestion of Burstein et al. ( $\left.{ }^{7}\right)$, superconductor tunnel junctions may be used for photon detection via breaking of Cooper pairs. Obviously, the corresponding process also applies for phonons of energy $E \geq 2 \Delta$.

A tunneling junction with two identical superconductors is biased in the voltage range of thermal quasiparticle tunneling $0<\mathrm{eV}<2 \Delta$ (see Fig. 2). Incident phonon radiation of energy $E \geq 2 \Delta$ will be strongly absorbed by breaking Cooper pairs, thereby increasing the number of excited quasiparticles. This results in a corresponding change of the tunneling current. Since only phonons of $E \geq 2 \Delta$ can contribute to pair breaking, detection has frequency-selective properties. Phonon absorption taking account of pair breaking has been calculated by Tewordt $\left(^{3}\right)$ and Bobetic $\left({ }^{13}\right)$. At $T=0$ phonon absorption disappears for phonon energies below $2 \triangle$ as predicted by BCS theory as well. At $2 \triangle$ the absorption increases discontinuously to a value of 1.57 times the normal-state absorption, and then with increasing energy monotonically approaches the normal-state value. At finite temperatures, e.g., $T / T_{c}=0.5$, this behaviour is not changed appreciably, the difference being a small absorption also for energies below

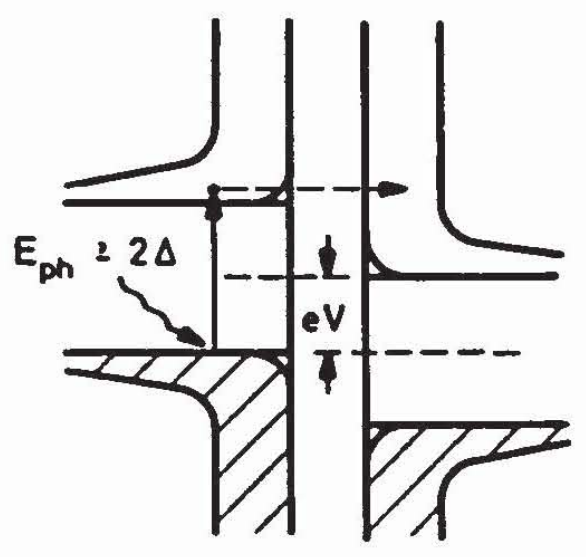

Fig. 2. Phonon detection, $0<e V<2 A$. 
$2 \triangle$ amounting to about 0.05 the normal-state value and a small shift in the position of the discontinuous absorption increase corresponding to the temperature dependence of the energy gap. Absorption at energies below 24 results from phonon interactions with thermally excited quasiparticles, and of course does not contribute to phonon detection. The same order of magnitude of phonon absorption via pair breaking and of normal-state absorption indicates that even with thin superconducting films used in a tunneling junction phonon detection with high sensitivity should be possible (compare the preceding estimate on the phonon mean free path in tin).

\section{EXPERIMENTAL PROCEDURE}

In the experiments reported in $\left.{ }^{14}\right)$ phonon generation and detection was performed using two identical $\mathrm{Sn}-I$-Sn junctions (strip width $1 \mathrm{~mm}$; thickness: bottom $1000 \AA$, top $3000 \AA$ ) evaporated on the optically polished end surfaces of a cylindrical sapphire rod of $1-\mathrm{cm}$ length and $1-\mathrm{cm}$ diameter. This arrangement (Fig. 3) allows for pulse measurements in order to check the phonon nature of observed signals by time-of-flight determination. A single crystal of synthetic sapphire with low dislocation density, $10^{2}-10^{3}$ $\mathrm{cm}^{-2}$, has been used because of the small phonon scattering by lattice imperfections it affords. It is further of importance that phonons generated

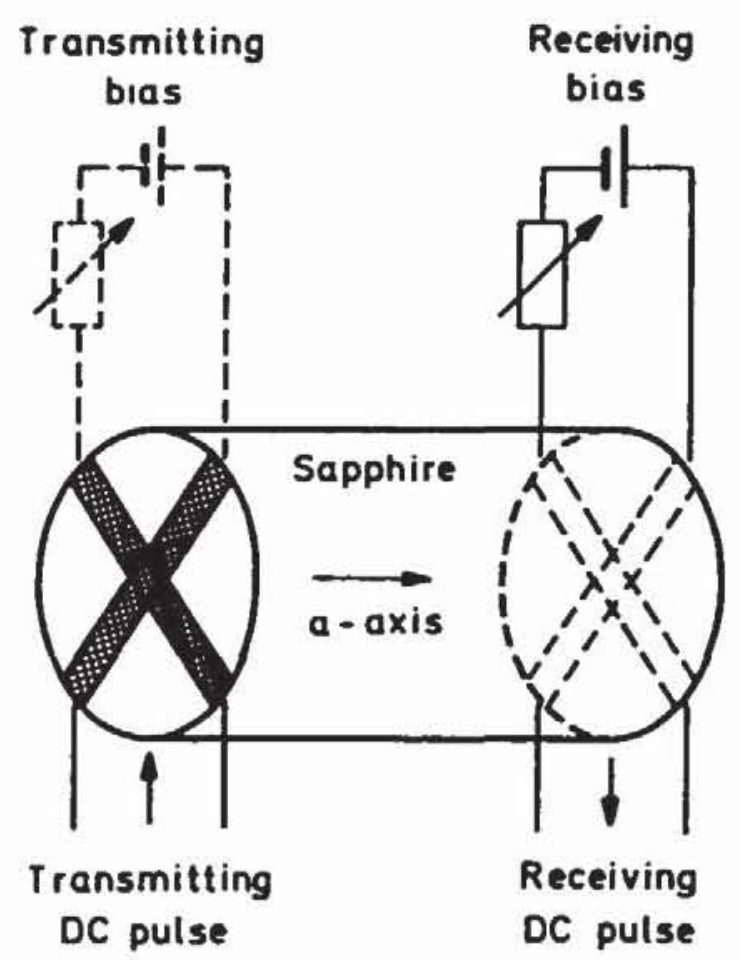

Fig. 3. Sapphire crystal with tunneling junctions. 
in the junction are transmitted to the sapphire with little reflection. An estimate of the acoustical mismatch at the tin-sapphire interface results in an energy transfer of about $85 \%$ for longitudinal and transverse phonons of normal incidence. The sample in Fig. 3 is in direct contact to liquid helium at temperatures of $1.2^{\circ} \mathrm{K}$.

Measurements of the DC $I-V$ characteristics of the junctions are performed in the familiar four-terminal technique. In a corresponding way it is also possible to obtain the characteristics under pulsed conditions.

For phonon propagation measurements DC current pulses with duration from 0.1 to $0.5 \mu \mathrm{sec}$ and a repetition rate of $10 \mathrm{kHz}$ are applied to the generating junction. In addition a variable DC bias can be superimposed. The detecting junction is operated with a current bias (high ohmic source) at a junction voltage of about $0.8 \mathrm{mV}$. Under these conditions detection results in voltage pulses at the free terminals of the junction. After lownoise wideband amplification the signals are fed to a sampling scope and finally plotted with the help of an $X-Y$ recorder (Fig. 4). Further noise reduction is accomplished by a low-pass filter at the $X-Y$ recorder input.

Since junctions with low ohmic tunneling resistance, typically 1-10 $\mathrm{m}$-ohm outside the gap region, are preferable as generator and detector, matching transformers are used with both junctions. A magnetic field parallel to the diode plane ranging from 10 to $15 \mathrm{G}$ serves to suppress Josephson DC currents and corresponding instabilities.

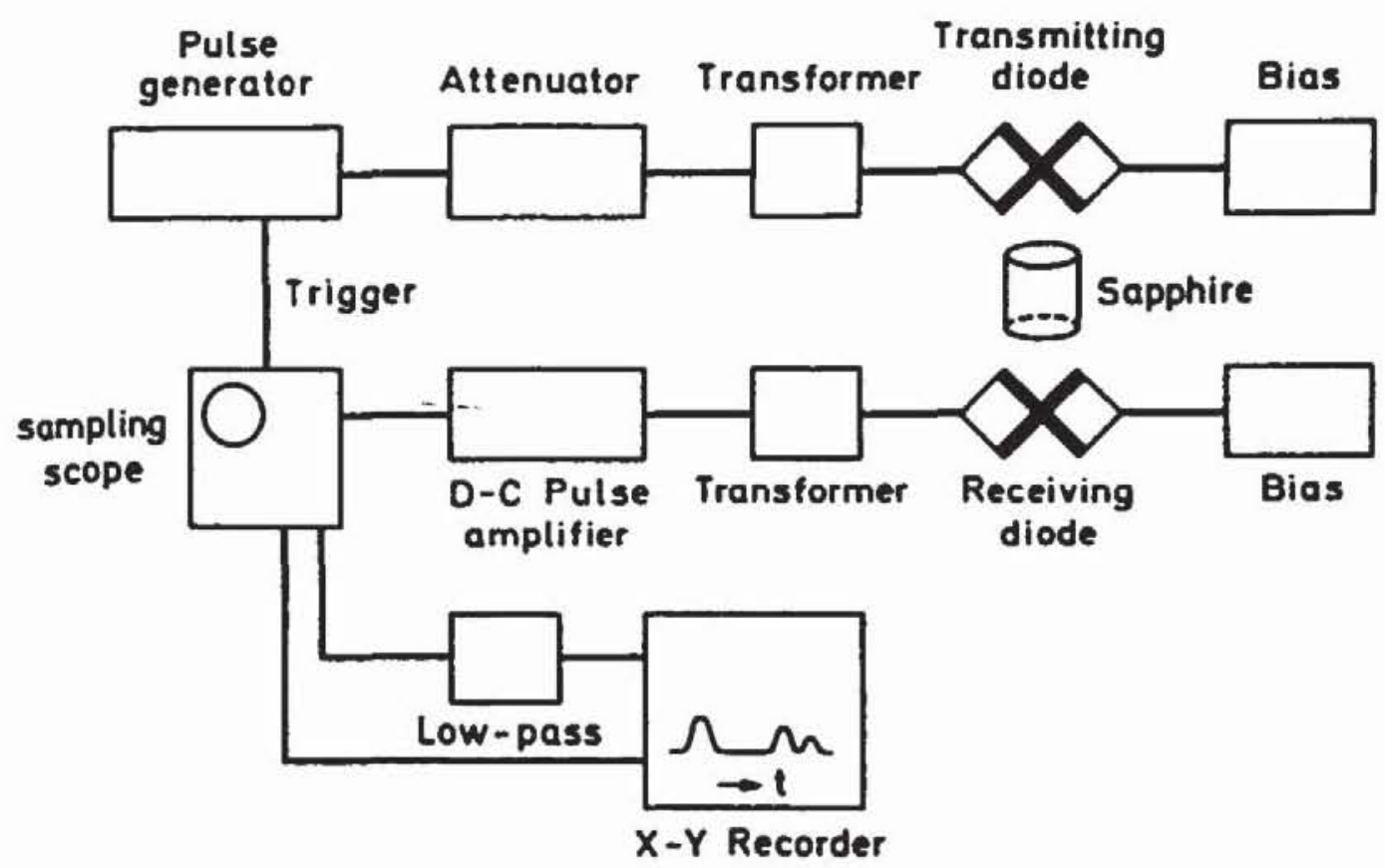

Fig. 4. Electronic setup. 


\section{EXPERIMENTAL RESULTS}

\section{Time-of-Flight Measurement}

Figure 5 shows phonon signals as function of time propagating parallel to the $a$ axis in sapphire. The first signal from the left corresponds to magnetic feedthrough and may serve as indication for zero delay. Subsequent signals correspond to the weak longitudinal mode and the strong fast transverse mode. The time delay of the last signal is too large to be attributed to a slow transverse mode; instead, diffuse scattering of the fast transverse mode at the fine-ground cylindrical sidewall of the sapphire crystal does account well for the observed time delay. Better time resolution is obtained at an elevated temperature of $1.9^{\circ} \mathrm{K}$ with an improved pulse system $\left({ }^{15}\right)$ (see Fig. 6). In this measurement $\mathrm{Pb}-I-\mathrm{Pb}$ was used for generation and $\mathrm{Sn}-I-\mathrm{Sn}$ for detection, the sapphire length in the $a$ direction amounting to $1.2 \mathrm{~cm}$. Time-of-flight evaluation for this measurement does agree with ultrasonic velocity data $\left.{ }^{(16}\right)$ in sapphire within $\pm 3 \%$ for all three modes in the $a$ direction. The slow transverse signal (weak) is now well separated from the strong fast transverse pulse and the sidewall scattering contribution. The shape and time delay of the sidewall scattering signal are in agreement with calculations using ultrasonic velocity data for the fast transverse mode assuming that scattering occurs only once for each phonon ray. An estimate of the corresponding signal amplitude in relation to the fast transverse signal amplitude indicates an omnidirectional or Lambert cosinus

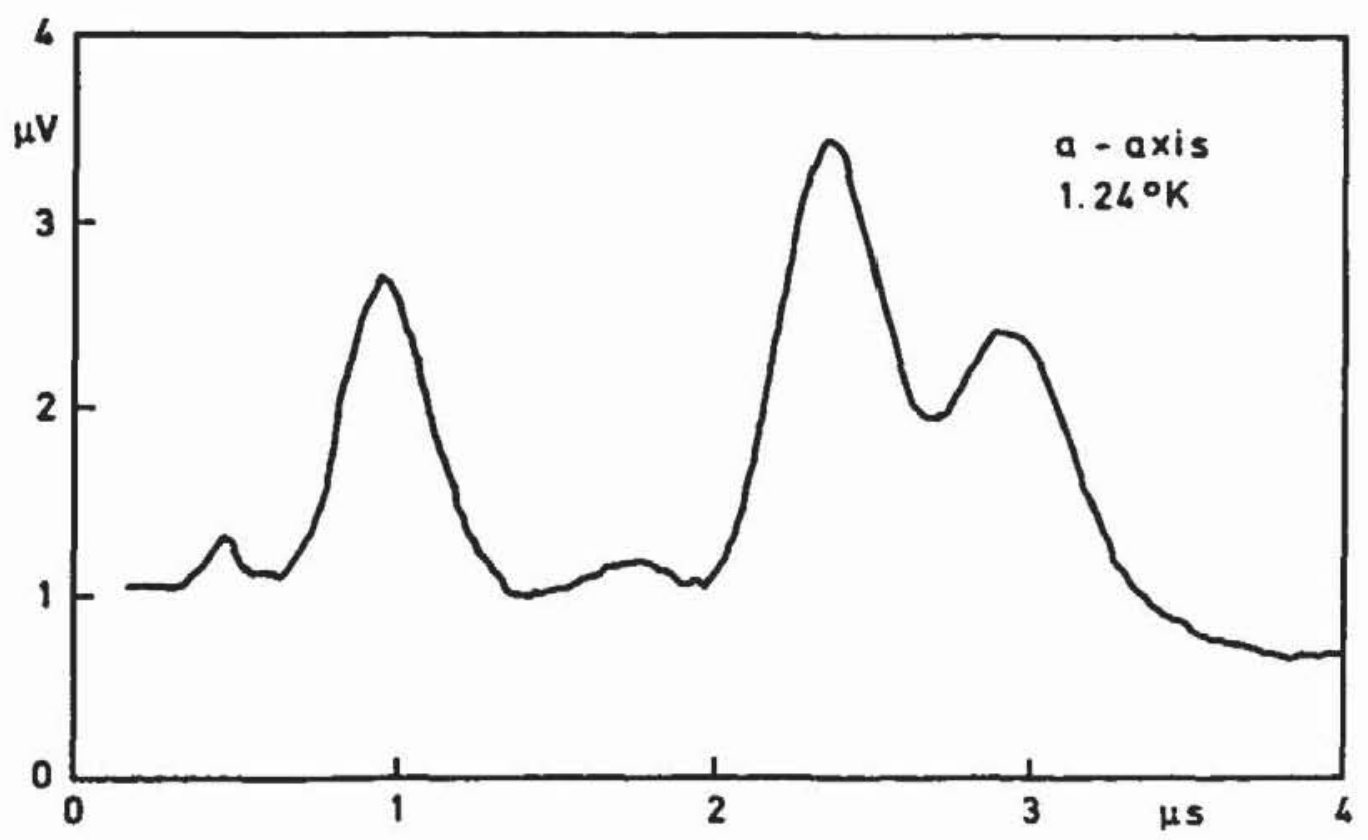

Fig. 5. Pulse signals, $a$ axis. Taken from Eisenmenger and Dayem (16). 


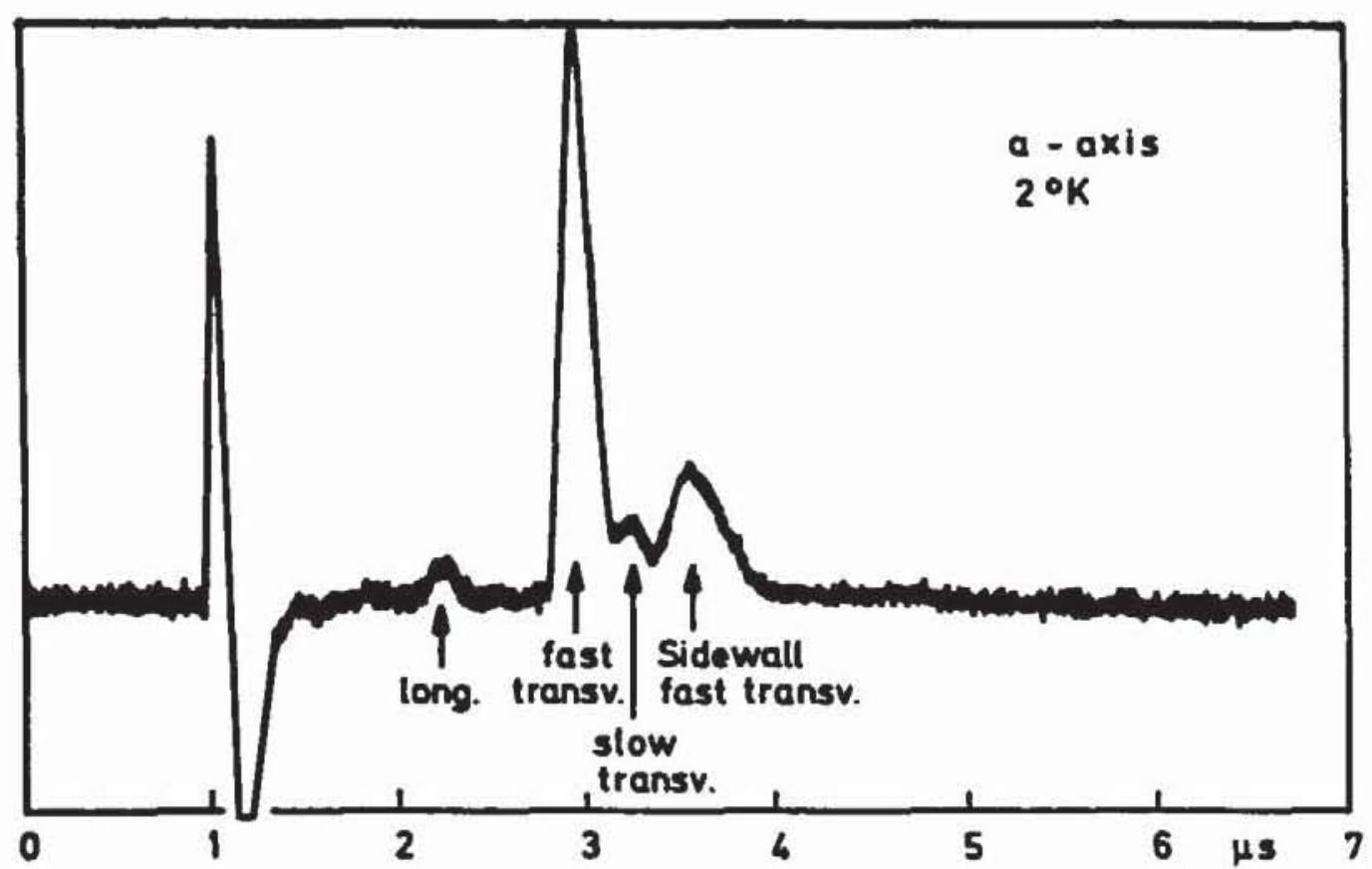

Fig. 6. Pulse signals, improved time resolution, $a$ axis.

law radiation distribution at the transmitter. This gives evidence for incoherent phonon emission from the generator. In corresponding pulse measurements for phonon propagation in $c$-axis sapphire (Fig. 7) only one transverse signal is observed, in agreement with the transverse wave degeneracy for this crystal orientation. Again, the time delay of the last

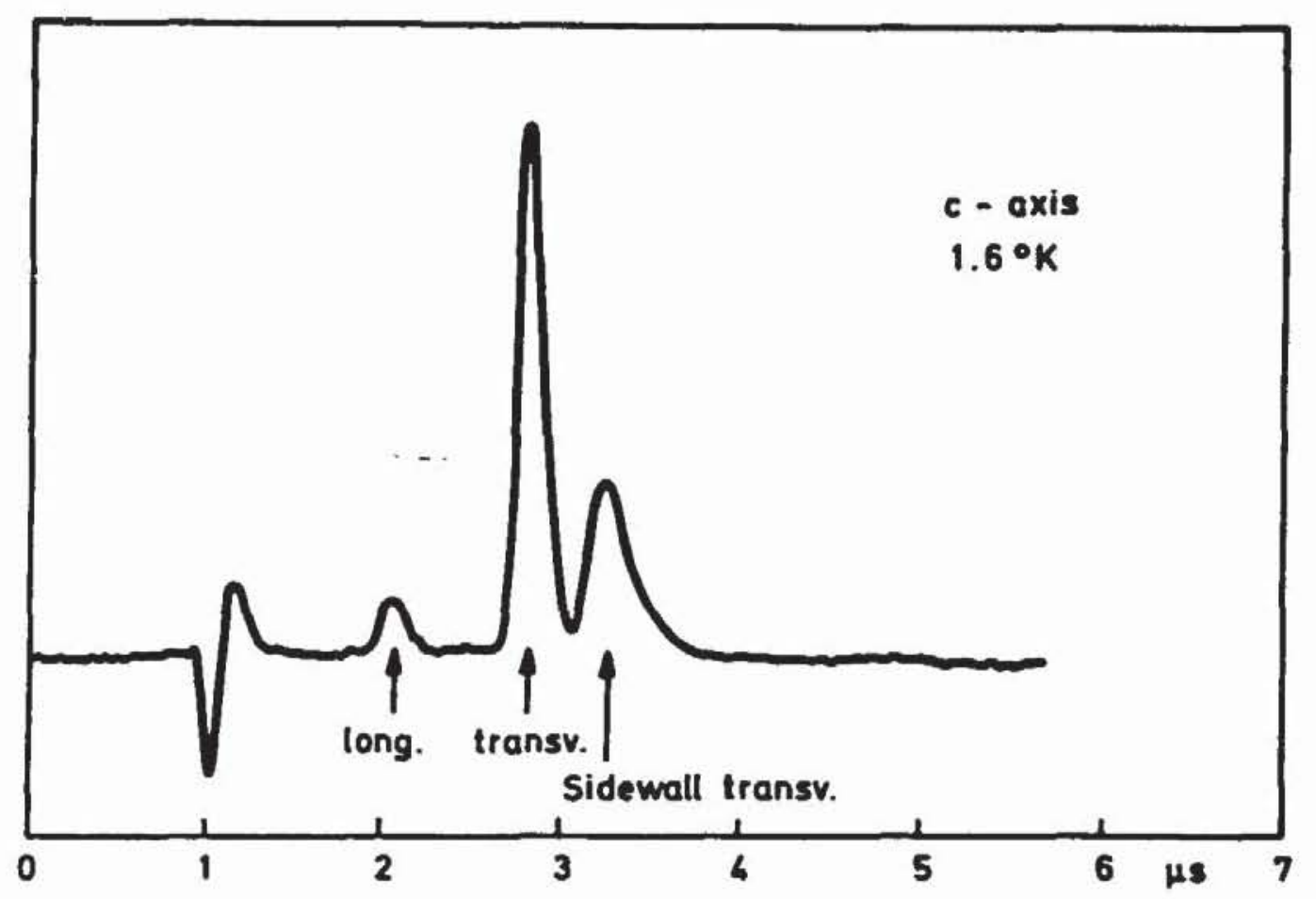

Fig. 7. Pulse signals, $c$ axis. 


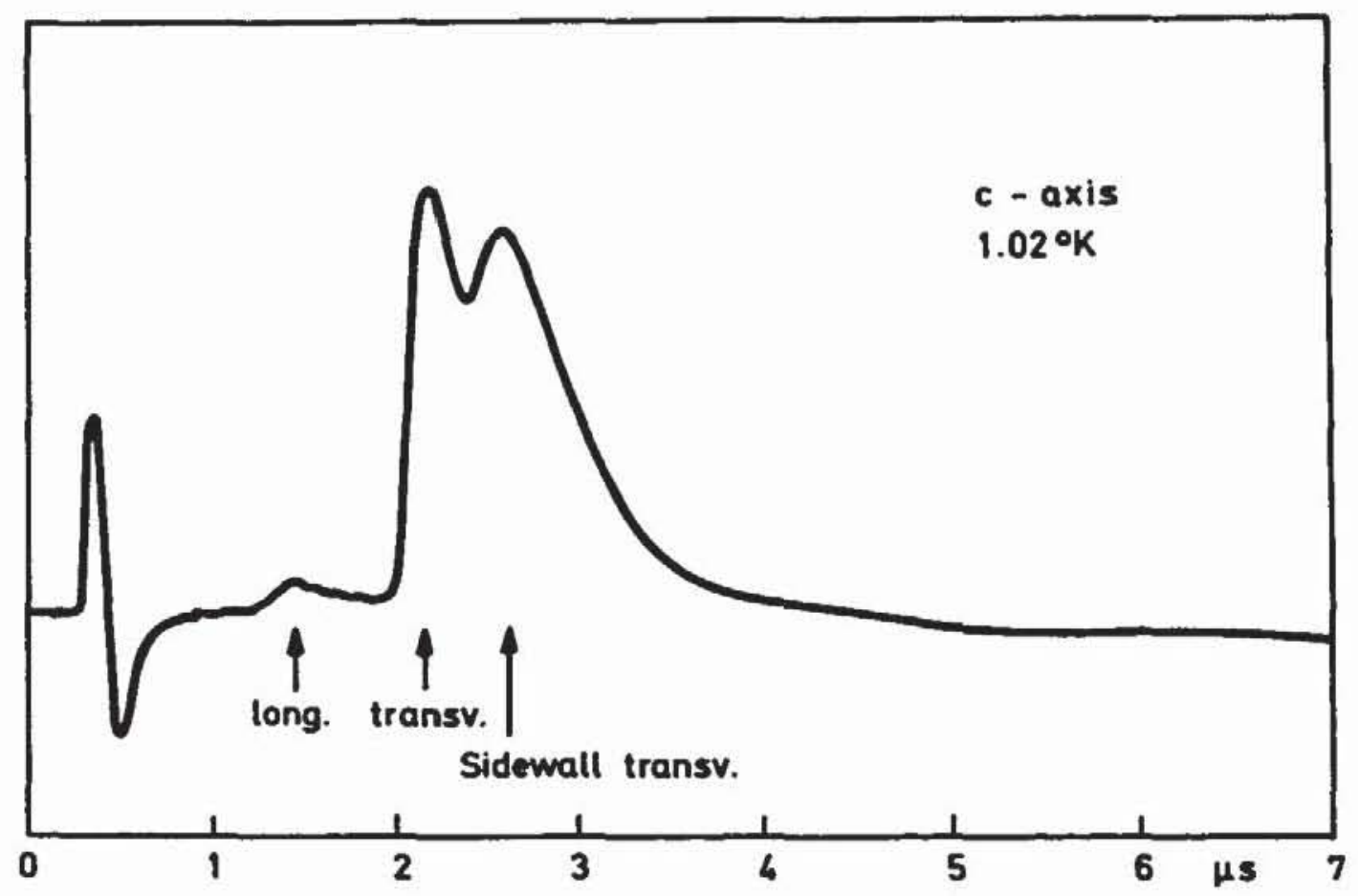

Fig. 8. Pulse signals, $c$ axis, low temperature.

signal resulting from sidewall scattering can be calculated to be in agreement with the scattering of the transverse wave.

In all pulse measurements there is an appreciable influence of temperature on pulse amplitude and time resolution. Lowering temperature results in increasing signal amplitude and detector time constant. Figure 8 shows signals obtained with the same sample as used in Fig. 7 but at a temperature of $1.02^{\circ} \mathrm{K}$. The marked increase in time constant indicated by slowly decaying pulse tails cannot be related to any temperature dependence of electrical circuit components. The temperature dependence of decay time and amplitude will be discussed later.

\section{Signal Amplitude as a Function of Generator Voltage}

Since phonon detection is possible only for phonon energies exceeding the gap energy, a voltage dependence of the generator phonon spectrum as expected for relaxation phonons can be checked by measurements of the signal amplitude as a function of generator pulse voltage or current amplitude. In Fig. 9 the detector signal amplitude (fast transverse wave) is plotted versus pulse current amplitude. Two identical $S n-I-S n$ junctions are used as generator and detector. In addition, the generator $I-V$ characteristic measured under pulse conditions is introduced using the same current scale. It is found that the signal amplitude first increases almost linearly with 


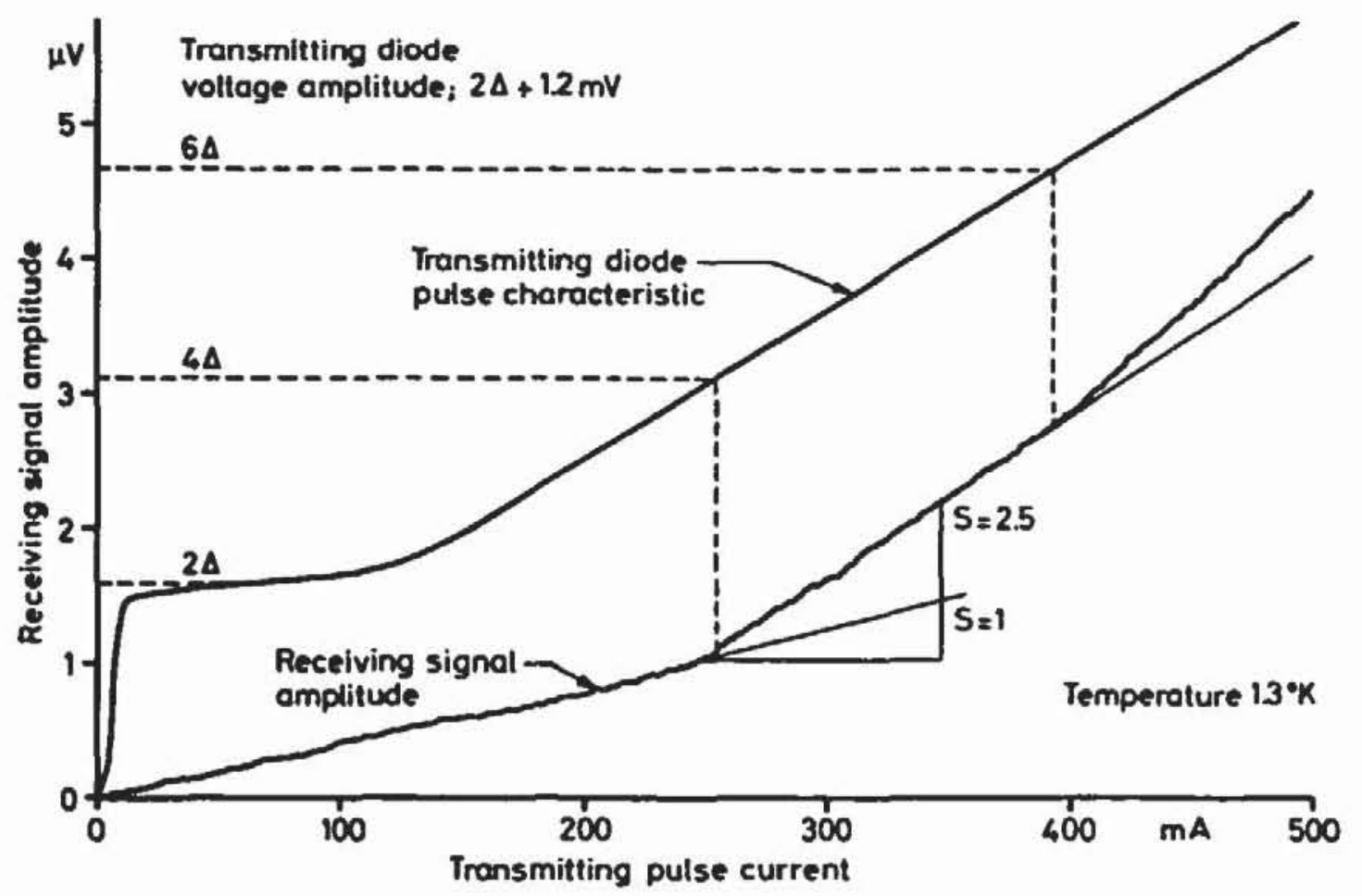

Fig. 9. Detector signal amplitude versus generator pulse current (voltage). Taken from Eisenmenger and Dayem ( ${ }^{(4)}$ ).

transmitter current. At a generator voltage corresponding to $4 \Delta$ in tin a sharp change in slope is succeeded again by a linear increase up to a generator voltage corresponding to 64 . From this point on an almost linear increase in slope is observed. This is more clearly brought out in the derivative with respect to generator current as shown in Fig. 10. To take the derivative a constant current pulse of $25 \mathrm{~mA}$ in addition to a variable DC bias current is applied to the generating junction. The signal in Fig. 10 is plotted versus bias current indicating junction voltages corresponding to $4 \Delta$ and $6 \Delta$. Since only pulse signals from the detector are amplified and recorded, the DC background of phonon radiation does not show up. In terms of the semiconductor model (Fig. 1) this experimental condition roughly corresponds to the injection of a constant number of quasiparticles in the range of maximum energy, the latter being determined by the biasdependent DC voltage drop across the junction.

For an interpretation of the findings shown in Fig. 9 and Fig. 10 we have now to remember the voltage dependence of the upper energy limit for relaxation phonons, $E_{\max }=e V-2 \Delta$, together with the condition that the detector is only sensitive to phonon energies larger than $2 A$. This implies that relaxation phonons do only contribute to the receiving signal if the generator voltage is larger than $4 \Delta / e$. In contrast, the contribution of re- 


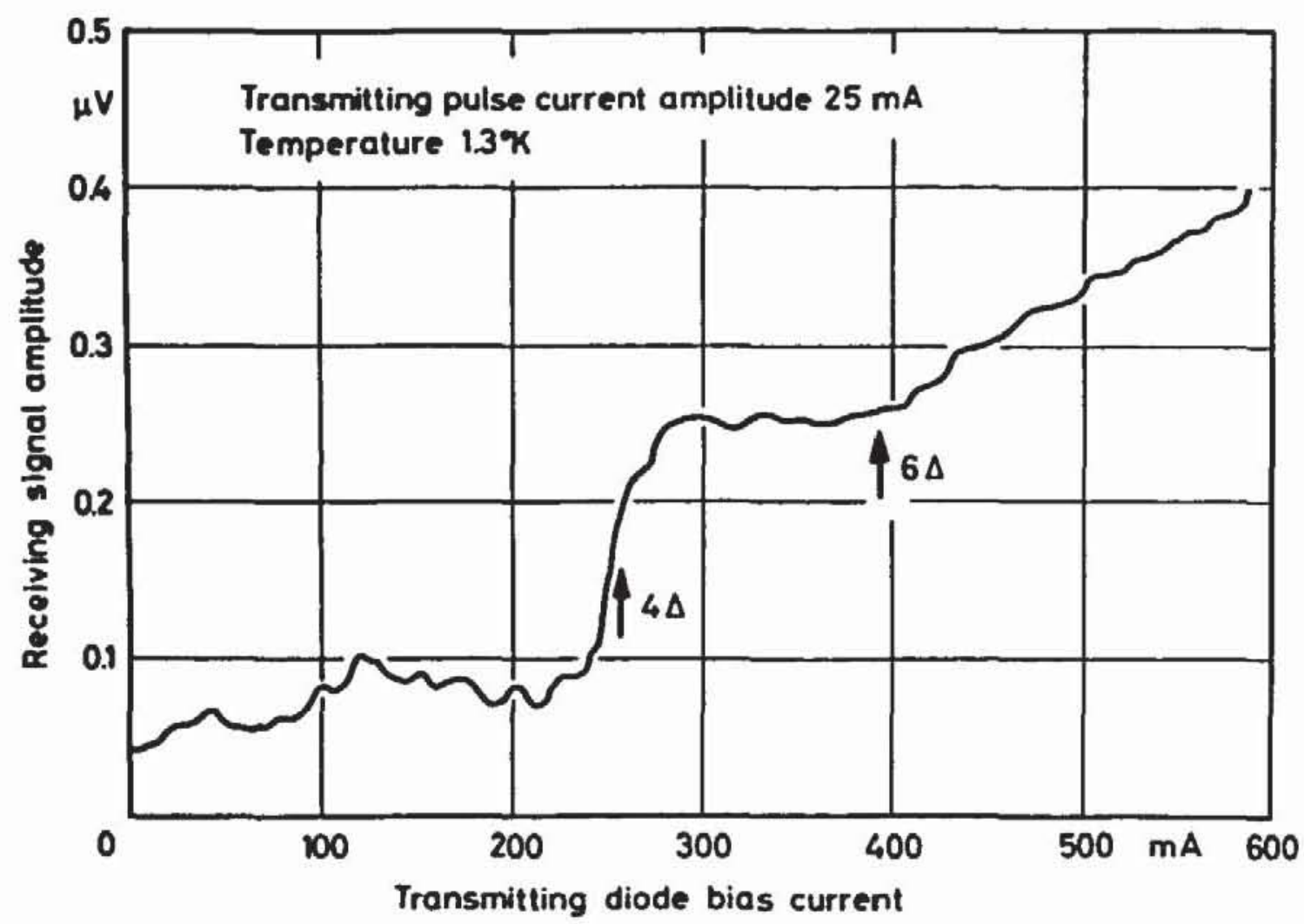

Fig. 10. Derivative of the detector signal of Fig. 9 plotted versus generator bias current (voltage) at constant generator pulse current. Taken from Eisenmenger and Dayem (")).

combination phonons with energies centered at $2 \Delta$ does not depend on the battery voltage. As long as $V$ is larger than $2 \Delta / e$,e. g., exceeding the range of thermally excited quasiparticle tunneling, the rate of recombination phonon emission is directly proportional to the single-particle tunneling current.

The changes in slope and in the step height at $4 \Delta$ in Fig. 9 and Fig. 10, respectively, are determined by the ratio of the number of relaxation to recombination phonons emitted. According to the semiconductor model (Fig. 1) we would expect this ratio to be 1 . More reliable conclusions might be drawn from the correct excitation diagram for tunneling between superconductors $\left({ }^{1,2}\right)$ under the condition of zero temperature and equal gap energies. For one electron to tunnel a Cooper pair must be broken up leaving one excitation on each side of the barrier with a total energy of $\mathrm{eV}$ referred to the ground state. The total energy with respect to the upper gap edges amounts to $e V-2 \Delta$. Correspondingly, in relaxation two phonons of total energy $e V-2 \Delta$ are emitted. The energies of the individual phonons can range from 0 to $e V-2 A$. In order to allow for recombination, a second Cooper pair must be broken up altogether, resulting in two excitations on each side. The decay of these excitations produces four relaxation phonons and two recombination phonons, resulting in a ratio of two to one. 
The same conclusion can be arrived at by simply considering energy conservation. If relaxation phonons have a continuous energy distribution between 0 and $e V-2 \Delta$, they must be emitted in pairs of total energy $e V$ $-2 \Delta$. This plus the recombination phonon energy $2 \Delta$ add up to the energy $\mathrm{eV}$ supplied in the tunneling of one electron.

Comparing the relaxation-recombination phonon ratio of two to one with the relative step height at $4 \Delta$ in Fig. 10, reasonable agreement is found.

In trying to explain the structure at $6 \Delta$ we find that this may be more involved than the simple interpretation applicable at $4 \Delta$. Basically, the $6 \Delta$ structure indicates the possibility of two phonons with energy $2 \Delta$ being emitted instead of one relaxation phonon with energy $4 \Delta$.

\section{Temperature Dependence of Signal Amplitude and Time Constant}

In discussing the effect of temperature variation we have to distinguish among properties of the phonon generator, the sapphire crystal, and the detecting junction.

The $I-V$ characteristic of superconductor tunneling junctions well below $T_{c}$ and with $\Delta$ approaching $\Delta_{0}$ essentially becomes temperature independent. An exception is to be made with respect to the voltage range $0<V<2 \Delta / e$, where the tunneling current is mainly determined by the number of thermally excited quasiparticles. Since this current is small compared to the tunneling current at voltages $V>2 \Delta / e$ (comprising in good junctions about $1 \%$ ), it can be neglected in phonon generation. Temperature changes in a range well below $T_{c}$ will therefore have little or no influence on the primary spectrum emitted in the relaxation and recombination of quasiparticles.

Phonon propagation inside the sapphire crystal may in principle be affected by temperature-dependent scattering of thermal lattice waves. Extrapolating ultrasonic absorption data $\left({ }^{(7)}\right)$ according to theory $\left({ }^{18}\right)$ to a frequency of $3 \times 10^{11} \mathrm{cps}$ and a temperature of $2^{\circ} \mathrm{K}$ results in a phonon mean free path of the fast transverse mode exceeding $10^{3} \mathrm{~cm}$. From this we expect that temperature-dependent phonon scattering in sapphire will be negligibly small, for instance, compared to temperature-independent scattering by lattice imperfections [compare also ${ }^{(11)}$ ].

Since experiment ${ }^{15}$ ) indicates a marked increase in signal amplitude with decreasing temperature (Fig. 11), this temperature dependence must be a property of the detection junction.

With incident phonons of energy $E \geq 2 \Delta$ being absorbed by breaking 


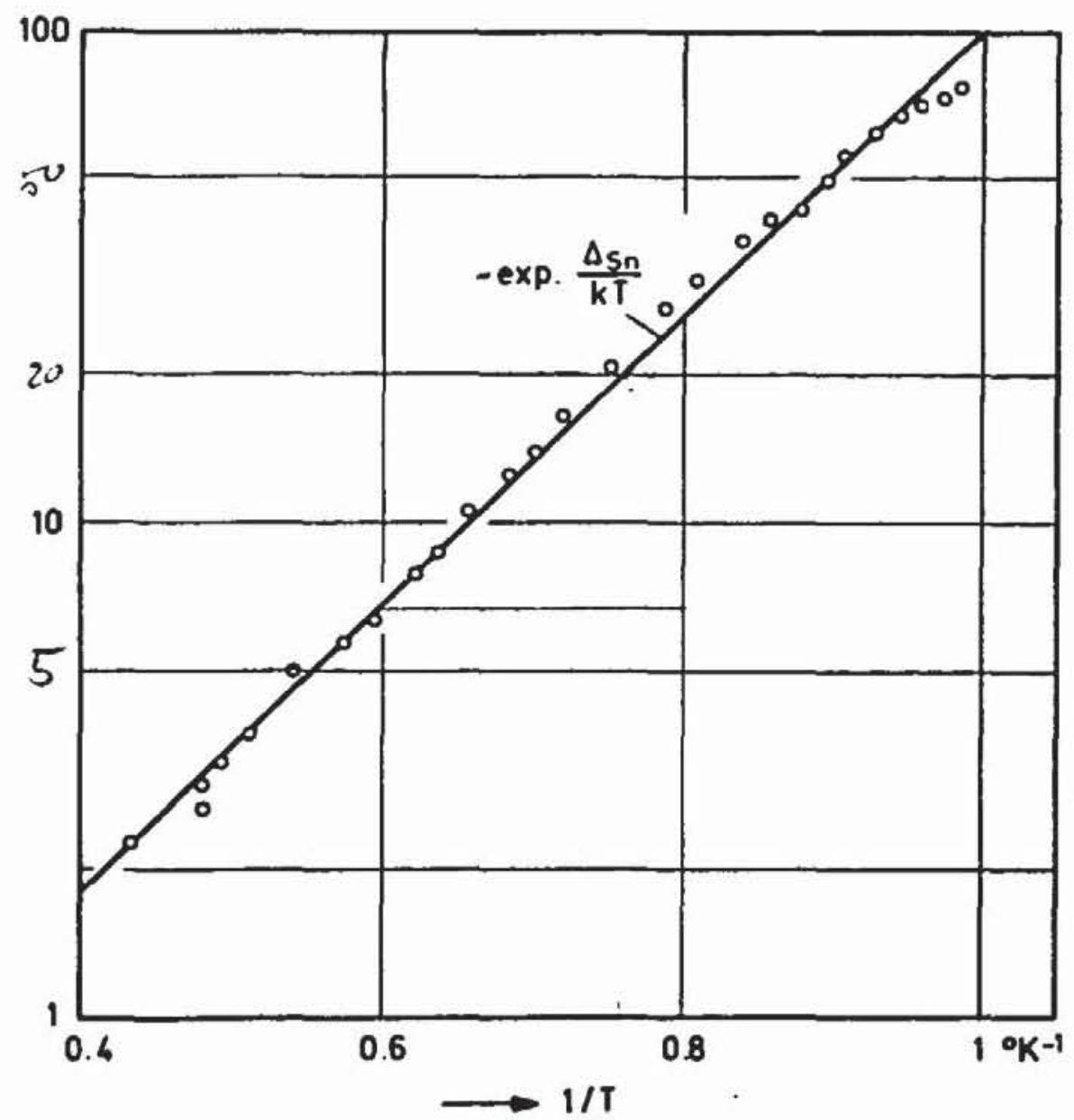

Fig. 11. Signal amplitude versus temperature, $\tau_{\text {pulse }}>\tau_{\text {eff }}$.

Cooper pairs in the detector, the steady-state increase from thermal equilibrium of the number of excited quasiparticles $\delta n$ amounts to

$$
\delta n=2 n_{\mathrm{ph}} \tau_{\mathrm{eff}},
$$

where $n_{\mathrm{ph}}$ corresponds to the number of absorbed phonons per unit time, each phonon creating two quasiparticles. According to $\left({ }^{13}\right)$, this absorption also shows little temperature dependence for $T / T_{c}<0.5$. Here $\tau_{\text {eff }}$ is the effective recombination lifetime of quasiparticles. The term "effective" indicates the possibility of reabsorption of phonons emitted by recombination in the detector [see also $\left({ }^{19}\right)$ ]. The steady-state expression is also valid for pulse measurements as long as $\tau_{\text {eff }}$ is small compared to the pulse duration. The receiver current $i_{R}$ (dynamical short-circuit current) is determined by the number of additional quasiparticles $\delta n$ per unit volume $v$ :

$$
\cdot i_{R}=(\delta n / v) C,
$$

where $C$ is a temperature-independent tunneling constant. Together with 
Eq. (1) this results in

$$
i_{R}=\tau_{\mathrm{eff}}\left(2 n_{\mathrm{ph}} / v\right) C,
$$

with $\tau_{\text {eff }}$ the only temperature-dependent factor.

Since $\tau_{\text {eff }}$ exceeds the recombination lifetime by only a constant factor, its temperature dependence is predicted $\left({ }^{6,19}\right)$ to be roughly proportional to $(1 / \sqrt{T}) e^{\Delta / R T}$ at low temperatures, or inversely proportional to the number of thermally excited quasiparticles. As shown in Fig. 11, for measurements with $\mathrm{Sn}-I-\mathrm{Sn}$ as detector, $i_{R}$ exhibits the expected temperature dependence.

In experiments with generator pulse duration comparable to $\tau_{\text {eff }}$ a corresponding time constant of the detector should show up directly in the pulse decay time. This has been observed $\left({ }^{15}\right)$ at low temperature (Fig. 8). Evaluations at different temperatures with respect to the absolute value of $\tau_{\text {eff }}$, taking account of the electronic system contribution to the pulse shape, are presented in Fig. 12. Again agreement with the expected temperature dependence of $\tau_{\text {eff }}$ is found. This result, together with the same temperature dependence of $i_{R}$, gives support to the assumptions made that

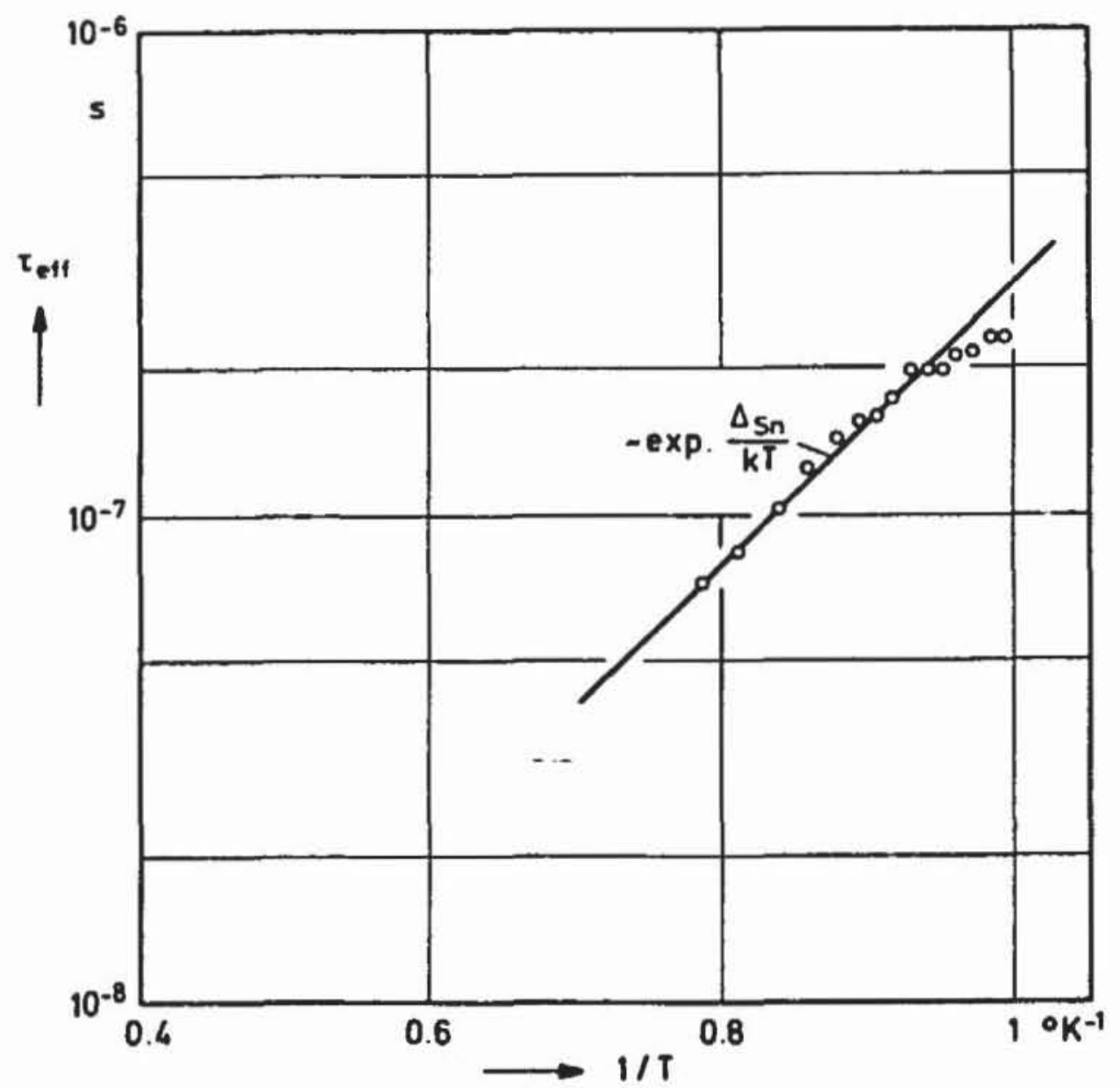

Fig. 12. Signal decay time (time constant) versus temperature. 
there are comparatively small temperature dependences of the generator phonon radiation, the phonon absorption inside the sapphire, and the phonon absorption in the detector.

\section{CONCLUSION}

In reviewing the experiments we should summarize the arguments indicating that the observed phonon signals are due to nonthermal phonon radiation emitted in the decay and recombination of injected quasiparticles.

It appears very difficult to explain the observed structure in the dependence of the signal amplitude as a function of the generator voltage in terms of heat pulses, e.g., phonons with Planck's distribution. An estimate of the possible heat effect arrives at much smaller signal amplitudes than observed in $\left({ }^{14}\right)$. In addition, only little temperature dependence of the signal amplitude is to be expected. In contrast to this, quantum processes provide a straightforward understanding of the experimental findings.

Finally, it might be worthwhile to mention that phonon-electron interactions, especially the phonon detection by pair breaking, indicate the close analogy to corresponding photon interactions as, e.g., in the photoelectric effect.

\section{REFERENCES}

1. J. R. Schrieffer, this volume, Chapter 21 .

2. J. Wilkins, this volume, Chapter 24.

3. L. Tewordt, Phys. Rev, 127:371 (1962).

4. L. Tewordt, Phys. Rev. 128:12 (1962).

5. B. I. Miller and A. H. Dayem, Phys. Rev. Letters 18:1000 (1967).

6. J. R. Schrieffer and D. M. Ginsberg, Phys. Rev. Letters 8:207 (1962).

7. E. Burstein, D. N. Langenberg, and B. N. Taylor, Phys. Rev. Letters 6:92 (1961).

8. W. P. Mason and H. Bömmel, Acoust. Soc. Am. 28:930 (1956).

9. W. P. Mason, Physical Acoustics and the Properties of Solids, D. Van Nostrand Inc., New York, 1958, p. 323ff.

10. R. Berman, E. L. Foster, and J. M. Ziman, Proc. Roy. Soc. (London) A231:130 (1955).

11. R. J. v. Gutfeld and A. H. Nethercot, Phys. Rev. Letters 12:641 (1964).

12. R. J. v. Gutfeld and A. H. Nethercot, Phys. Rev. Letters 17:868 (1966).

13. V. M. Bobetic, Phys. Rev. 136:A1535 (1964).

14. W. Eisenmenger and A. H. Dayem, Phys. Rev. Letters 18:125 (1967).

15. W. Eisenmenger, A. H. Dayem, and B. I. Miller, unpublished work.

16. B. T. Bernstein, J. Appl. Phys. 34:169 (1963).

17. J. de Klerk, Phys. Rev. 139:A1635 (1965).

18. L. Landau and G. Rumer, Phys. Z. Sowjetunion 11:18 (1937).

19. A. Rothwarf and B. N. Taylor, Phys. Rev. Letters 19:27 (1967). 